\title{
Single-Port-Access-Cholezystektomie als sichere Alternative zum 4-Port-Vorgehen: Ein retrospektiver Vergleich
}

\author{
Single-Port Access Cholecystectomy is a Safe Alternative to Conventional Laparoscopic Cholecystectomy: \\ A Retrospective Comparison of Single-Port Access versus Standard Laparoscopic Cholecystectomy
}

Autoren

Institute

\section{K. Cziupka' ${ }^{1}$ L. I. Partecke², C. Nass ${ }^{1}$, L. Mirow}

${ }^{1}$ Landkreis Mittweida Krankenhaus gGmbH, Allgemein- und Viszeralchirurgie, Mittweida, Deutschland

${ }^{2}$ Universität Greifswald, Chirurgie, Greifswald, Deutschland

\section{Schlüsselwörter \\ - Abdominalchirurgie \\ - laparoskopische Chirurgie \\ - chirurgische Technik}

Key words

- abdominal surgery

- laparoscopic surgery

- surgical technique
Bibliografie

DOI http://dx.doi.org/

$10.1055 / \mathrm{s}-0031-1283886$

Online-Publikation: 16.3.2012

Zentralbl Chir 2013; 138: 70-75

(c) Georg Thieme Verlag KG

Stuttgart · New York

ISSN 0044-409X

Korrespondenzadresse

PD Dr. Lutz Mirow

Landkreis Mittweida

Krankenhaus gGmbH

Hainicher Str. 4-6

09648 Mittweida

Deutschland

Tel.: 03727 / 991240

Fax: 03727 / 991445

lutz.mirow@lmkgmbh.de

\section{Zusammenfassung}

\section{$\nabla$}

Einleitung: Mit der Weiterentwicklung der laparoskopischen Chirurgie ist ein Ziel, Narben und operatives Trauma auf ein Minimum zu reduzieren. Die Single-Port-Chirurgie erfolgt über lediglich eine einzige Inzision in der Nabelgrube. Wir berichten über unsere Erfahrungen mit 69 SinglePort-Cholezystektomien im Vergleich zum Standard-4-Port Vorgehen.

Methode: Innerhalb von zwei Jahren führten wir 69 Single-Port-Cholezystektomien durch. Diese wurden in einer Fall-Kontroll-Studie mit in diesem Zeitraum durchgeführten 4-Port-Cholezystektomien verglichen. Dazu wurden demografische Daten der Patienten, Schnitt-Naht-Zeiten, postoperative Krankenhausaufenthaltsdauer, intra- und postoperative Komplikationen sowie Konversionsraten retrospektiv erfasst.

Ergebnisse: Zwischen der Single-Port-Methode und der Standard-4-Port-Cholezystektomie ergaben sich keine Unterschiede hinsichtlich der Rate intra- oder postoperativer Komplikationen. Die Schnitt-Naht-Zeiten waren abhängig vom Operateur und unterschieden sich zwischen beiden Methoden nicht. Die postoperative Krankenhausaufenthaltsdauer war tendenziell in der SinglePort-Gruppe kürzer, dies war jedoch statistisch nicht signifikant.

Schlussfolgerung: In ausgewählten Patientengruppen ist die Single-Port-Cholezystektomie dem Standard-4-Port-Vorgehen ebenbürtig. Der kosmetische Vorteil wird dabei nicht mit einem Verlust von Patientensicherheit erkauft.

\section{Einleitung}

\section{$\checkmark$}

Nachdem Erich Mühe im Jahre 1985 die erste laparoskopische Cholezystektomie durchführte [1], hat die Chirurgie des biliären Systems eine Revolution erlebt. Heute - nur etwas mehr als 25 Jahre später ist das laparoskopische Vorgehen als Standard bei

\section{Abstract \\ $\nabla$}

Purpose: As laparoscopic surgery develops, one current goal is the reduction of scarring and operative trauma to a minimum. Single-port access surgery (SPA) uses a single small incision hidden in the umbilicus. This report describes our experiences with 69SPA cholecystectomies compared to the conventional laparoscopic approach.

Methods: Within two years 69 SPA cholecystectomies were performed. Data including demographic distribution, incision to closure time, duration of the postoperative hospital stay, rates of intraoperative and postoperative complications, and conversion rates were collected retrospectively. Data for a matched control group treated by standard laparoscopic cholecystectomy were gathered within the same time frame and then compared.

Results: There were no significant differences in the rates of intraoperative or postoperative complications in the SPA group when compared to the standard laparoscopy group. The incision-to-closure time strongly depended on the surgeon but did not depend on the method. The duration of postoperative hospital stay was slightly decreased in the SPA group. However, this effect was statistically not significant.

Conclusions: For selected patient groups, SPA cholecystectomy is on par with the conventional laparoscopic method. The cosmetic advantage that it offers does not come at the cost of any loss of safety.

gutartigen Erkrankungen des biliären Systems akzeptiert und kann als eine der größten Errungenschaften der letzten zwanzig Jahre in der operativen Medizin bezeichnet werden. Lange stehen nicht mehr nur die kosmetischen Vorteile der Laparoskopie im Vordergrund, sondern vielmehr die kürzeren Rekonvaleszenzzeiten mit einer vermin- 
derten Krankenhausverweildauer, der niedrigere Schmerzmittelbedarf sowie die niedrigere Inzidenz an Narbenhernien [2]. Diese Entwicklung ist umso erstaunlicher, als anfänglich die Komplikationen bei der laparoskopischen Cholezystektomie bedeutend höher waren als die bei der offenen Cholezystektomie. Die weitere Minimalisierung des Traumas des operativen Zugangs war auch in den letzten Jahren eine enorme Bestrebung in der operativen Medizin. Der Wunsch nach „narbenloser Chirurgie“ schien sich im NOTES-Konzept zu erfüllen. Marescaux berichtete als erster von einer transvaginalen Cholezystektomie bei einer 30-jährigen Frau [3]. Allerdings wurde bezüglich dieses Konzepts die Frage geäußert, ob der transvaginale Zugang nicht das Risiko der Dyspareunie, Infertilität oder Versprengung einer bestehenden Endometriose berge [4]. Diese Frage lässt sich aufgrund des Fehlens von Langzeitergebnissen nicht abschließend beantworten. Eine Alternative zu NOTES stellt die „single port access (SPA)“ Laparoskopie dar [5]. Nach den ersten Veröffentlichungen einer SPA-Cholezystektomie durch Navarra 1997 [6] und Piskus im Jahre 1999 [7] erschienen ein Jahrzehnt lang kaum weitere Berichte in der Literatur. Seit dem Jahre 2008 folgten dann wieder viele Publikationen zu diesem „nahezu narbenlosen“ Vorgehen in der Weltliteratur, welche dieses Vorgehen überwiegend als sichere Alternative mit lediglich einer Narbe, welche in der Nabelgrube verschwindet, darstellen [810]. Dementsprechend favorisierten in einer Umfrage unter der weiblichen Bevölkerung die Mehrzahl auch das SPA-Vorgehen vor NOTES [11].

Die überwiegende Mehrzahl der Berichte über die SPA-Cholezystektomie sind Einzelfallberichte oder Studien ohne Vergleichsgruppe. Weiter existieren Metaanalysen [9] und Multizenterstudien [12]. Des Weiteren gibt es einige randomisierte Studien, welche die SPA-Cholezystektomie mit der 4-Trokar-Methode vergleichen [13-18]. Dabei zeigte sich in den meisten Studien eine höhere kosmetische Zufriedenheit und teilweise auch ein verminderter Schmerzmittelbedarf bei der SPA-Methode auf Kosten längerer Schnitt-Naht-Zeiten. Wir berichten in dieser Arbeit von den ersten 69SPA-Cholezystektomien an einem Haus der Regelversorgung und vergleichen diese mit dem konventionell-laparoskopischen Vorgehen.

\section{Material und Methoden}

$\nabla$

\section{Datenakquise}

Es erfolgte eine retrospektive Datenanalyse aller Standard4-Port- und SPA-Cholezystektomien, welche vom 1. Januar 2009 bis 31. Dezember 2010 in Mittweida operiert wurden. Erfasst wurden Geschlecht, Geburtsdatum, Operationsdatum, SchnittNaht-Zeiten, perioperative Mortalität, postoperative Krankenhausverweildauer, intraoperative Komplikationen, postoperative Komplikationen, welche bis zum Januar 2011 auftraten, ASA-Stadium, abdominelle Voroperationen, Einweisungsmodus (Notfall versus geplant), Histologie, die Rate an Patienten, bei welchen intraoperativ eine Drainage eingelegt wurde, sowie die Konversionsrate zum offenen Vorgehen. Für die SPA-Cholezystektomie wurde zudem die Rate an Extrainzisionen für eine Drainage sowie die Notwendigkeit eines zusätzlichen Portes und die Rate an Konversion zum konventionell-laparoskopischen Vorgehen erfasst.

\section{SPA-Cholezystektomie}

Die Patienten wurden im Vorfeld über die SPA-Methode und ihre Komplikationsmöglichkeiten ausführlich aufgeklärt. Die SPACholezystektomie wurde mit dem Triport $^{\circledR}$ System der Firma
Olympus sowie dem Gelpoint ${ }^{\circledR}$ der Firma Applied Medical durchgeführt. Die Operationen erfolgten in Intubationsnarkose in Steinschnittlage. Der Operateur steht zwischen den Beinen des Patienten und der Assistent sowie die instrumentierende Schwester an der linken Patientenseite. Nach Abwaschen und Abdecken erfolgt eine etwa $1 \mathrm{~cm}$ lange Inzision bogenförmig entlang der unteren Zirkumferenz der Nabelgrube. Die Linea alba wird dargestellt und Faszie und Peritoneum über etwa $1 \mathrm{~cm}$ längs inzidiert. Nach digitaler Austastung nach eventuellen Verwachsungen wird das entsprechende Portsystem über einem Wundretraktor eingebracht und das Pneumoperitoneum angelegt. Die Kamera wird über den zentralen 10-mm-Port eingebracht und die beiden 5-mm-Arbeitskanäle in 2-Uhr- und 8-Uhr-Position eingebracht. Die Präparation erfolgt mit bipolarer Schere, Ductus cysticus und A. cystica werden mit Clips versorgt. Der Verschluss von Faszie und Peritoneum erfolgt durch durchgreifende Einzelknopfnähte, der Verschluss der Haut mit Einzelknopfnähten nach Donati.

\section{Laparoskopische Cholezystektomie}

Der Zugang erfolgt bei der laparoskopischen Cholezystektomie infraumbilikal via Minilaparotomie. Zusätzlich werden ein 10mm-Trokar epigastrisch und zwei 5-mm-Trokare im rechten Oberbauch und Unterbauch eingebracht. Die Präparation erfolgt auch hier mittels bipolarer Schere. Ductus cysticus und A. cystica werden zwischen Clips durchtrennt.

\section{Datenanalyse}

Die SPA-Cholezystektomien wurden von vier laparoskopisch erfahrenen Operateuren durchgeführt. In der 4-Port-Gruppe wurden lediglich Patienten eingeschlossen, welche ebenfalls von diesen vier Operateuren operiert worden waren. Patienten, welche notfallmäßig operiert wurden, wurden ausgeschlossen.

Wir führten eine retrospektive Fall-Kontroll-Analyse durch. Die Datenanalyse erfolgte mittels SPSS Version 18.0 für Mac OS.Die Daten wurden auf eine Normalverteilung überprüft. P-Werte wurden mittels des t-Testes für normal verteilte Daten sowie des Mann-Whitney-U-Testes für nicht-normal verteilte Daten ermittelt. Nominale Daten wurden mittels Chi-Quadrat-Test verglichen. Für alle Analysen wurde ein Signifikanzniveau von 0,05 als signifikant festgelegt. Die Ergebnisse werden hier als Mittelwert \pm Standardfehler des Mittelwertes angegeben.

\section{Ergebnisse \\ $\nabla$}

Vom 1.Januar 2009 bis zum 31.Dezember 2010 wurden $69 \mathrm{~Pa}-$ tienten in SPA-Technik cholezystektomiert. 59,4\% $(n=41)$ davon wurden mit dem Gelpoint ${ }^{\circledR}-$ System der Firma Applied operiert, $40,6 \%(n=28)$ mit dem Triport ${ }^{\circledR}$ der Firma Olympus. Alle Patienten wurden elektiv aufgrund von chronischer Cholezystitis oder symptomatischer Cholezystolithiasis aufgenommen. Die Operationen wurden von fünf laparoskopisch erfahrenen Chirurgen durchgeführt. Das durchschnittliche Patientenalter in der Single-Port-Gruppe betrug 52,4 $\pm 1,7$ Jahre (14 bis 78 Jahre). Die mittels Standard-4-Port-Laparoskopie operierten Patienten waren im Durchschnitt $54,8 \pm 1,8$ Jahre alt ( 15 bis 83 Jahre). Somit ergeben sich keine signifikanten Unterschiede zwischen den beiden Gruppen ( $\mathrm{p}=0,3)$.

Auch hinsichtlich der Verteilung bezüglich das ASA-Stadiums unterscheiden sich die beiden Gruppen nicht signifikant $(p=0,08$; - Tab. 1). 
Intraabdominelle Voroperationen stellten keine Kontraindikation für das Single-Port-Vorgehen dar: So waren in der Single-PortGruppe 8,7\% ( $\mathrm{n}=6)$ abdominell voroperiert, in der Standard-Laparoskopie-Gruppe betrug die Rate 7,2\% $(n=5)$ ( $\bullet$ Tab.1). Hinsichtlich der Geschlechterverteilung ist der Anteil weiblicher Patientinnen signifikant höher in der Single-Port-Gruppe (54,7\% versus $45,3 \%, \mathrm{p}=0,04)$.

Die mittlere Schnitt-Naht-Zeit betrug 58,8 $\pm 3,0$ Minuten (20 bis $135 \mathrm{~min}$ ) für die Single-Port-Cholezystektomie, während die mittlere Schnitt-Naht-Zeit für die Standard-laparoskopisch operierte Gruppe 70,2 $\pm 2,9$ Minuten betrug ( $40 \mathrm{~min}$ bis $149 \mathrm{~min}$ ). Betrachtet man jedoch die Schnitt-Naht-Zeiten für jeden Operateur einzeln, so ergeben sich keine Unterschiede zwischen Standard4-Port-Verfahren und SPA-Technik ( $\bullet$ Abb.1). Innerhalb der Single-Port-Gruppe nahmen die Schnitt-Naht-Zeiten mit zunehmender Expertise des Operateurs ab. A Abbildung 2 zeigt die Lernkurve für alle Operateure: Dabei zeigt sich in allen Fällen eine Abnahme der Schnitt-Naht-Zeit während der ersten zehn Operationen. Nach zehn Single-Port-Cholezystektomien wird ein Plateau erreicht. Die Schnitt-Naht-Zeiten waren unabhängig vom verwendeten System: So betrug die mittlere Schnitt-NahtZeit bei Verwendung des Triport $^{\circledR}$ von Olympus $57,5 \pm 4,4 \mathrm{~min}$ und bei Verwendung des Gelpoint ${ }^{\circledR}$ von Applied $59,7 \pm 4,1 \mathrm{~min}$ $(\mathrm{p}=0,722)$.

Patienten, welche in Single-Port-Technik cholezystektomiert wurden, hatten eine durchschnittliche Krankenhausverweildauer von 5,25 $\pm 0,29$ Tagen, während die Patienten, welche in 4Port-Technik operiert wurden, durchschnittlich 5,8 $\pm 0,35$ Tage stationär behandelt wurden. Dieser Trend war statistisch jedoch nicht signifikant $(\mathrm{p}=0,1)$.

In der Single-Port-Gruppe benötigten 18,2\% aller Patienten eine zusätzlich Inzision für die Einbringung einer Drainage, während bei der Standard-4-Port-Technik immer eine Drainage eingelegt wurde. Die routinemäßige Einlage einer Drainage in das Gallenblasenbett im Rahmen einer 4-Port-Cholezystektomie entspricht einem internen Standard in der Landkreis Mittweida Krankenhaus gGmbH ohne Evidenz.

Zu einer akzidentiellen Gallenblasenperforation kam es in 11,5\% (8 Fälle) der 4-Port-Cholezystektomien, während die Rate in der SPA-Gruppe nur 7,2\% (5 Fälle) betrug (Chi-Quadrat 11,3, p= 0,004).

In keinem unserer Fälle war eine Konversion zum offenen Vorgehen notwendig. Zur Vermeidung eines Majorfehlers war in acht Fällen $(11,5 \%)$ in der Single-Port-Gruppe die Einbringung eines zusätzlichen Trokars notwendig. Nur in einem Fall war eine Konversion vom Single-Port-Verfahren zum 4-Port-Vorgehen notwendig. Hierbei handelte es sich um eine chronische Cholezystitis mit Verschwartung im Calotschen Dreieck.

In der 4-Port-Gruppe betrug die Rate intraoperativer Komplikationen 5,8\% ( $\mathrm{n}=4)(\bullet$ Tab.2). In drei Fällen handelte es sich dabei um eine Blutung aus dem Leberbett, welche mittels Koagulation gestillt werden konnte. In einem weiteren Fall kam es zu einer Blutung aus dem Omentum majus, welche intraoperativ mittels Clip sicher versorgt werden konnte. In der Single-PortGruppe unterschied sich die Rate intraoperativer Komplikationen $(\mathrm{n}=3)$ nicht signifikant von der 4-Port-Gruppe. In allen drei Fällen handelte es sich dabei um eine Blutung aus dem Leberbett, welche ebenfalls mittels Elektrokoagulation versorgt wurde ( $\bullet$ Tab.2).

7 Patienten aus der Single-Port-Gruppe erlitten postoperative Komplikationen ( Tab.3). Davon waren vier Komplikationen chirurgische Komplikationen. Diese beinhalteten eine Zystikus-

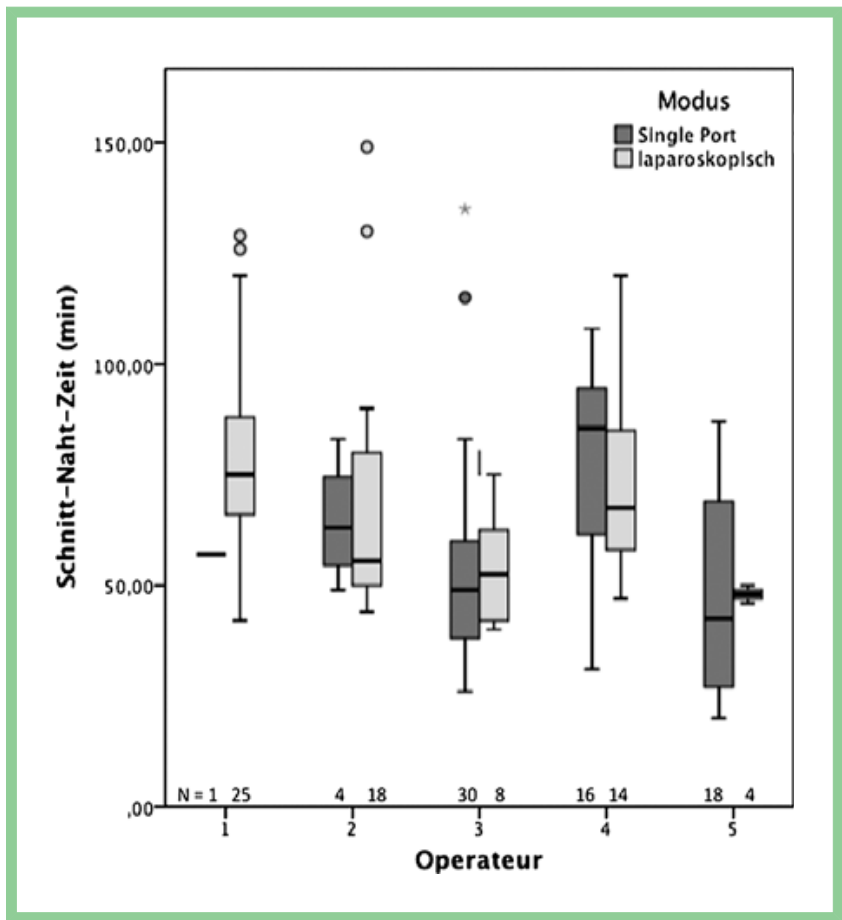

Abb.1 Schnitt-Naht-Zeiten, aufgeschlüsselt nach Operateur. Dargestellt sind Boxplots mit Median und 95\%-Konfidenzinterval. Ausreißer sind als Kreise dargestellt, extreme Ausreißer als Sternchen. Die hell gefärbten Balken-Box-Plots stellen die Schnitt-Naht-Zeiten beim 4-Port-Vorgehen dar, die dunklen Balken repräsentieren die Schnitt-Naht-Zeiten für die SinglePort-Cholezystektomie. Weiterhin ist die Anzahl der in dem beobachteten Zeitraum durchgeführten Eingriffe für jeden Operateur $(\mathrm{N})$ angegeben. Zwischen den beiden Methoden bestehen keine Unterschiede für jeden Operateur, allerdings sind die Schnitt-Naht-Zeiten vom Operateur abhängig.

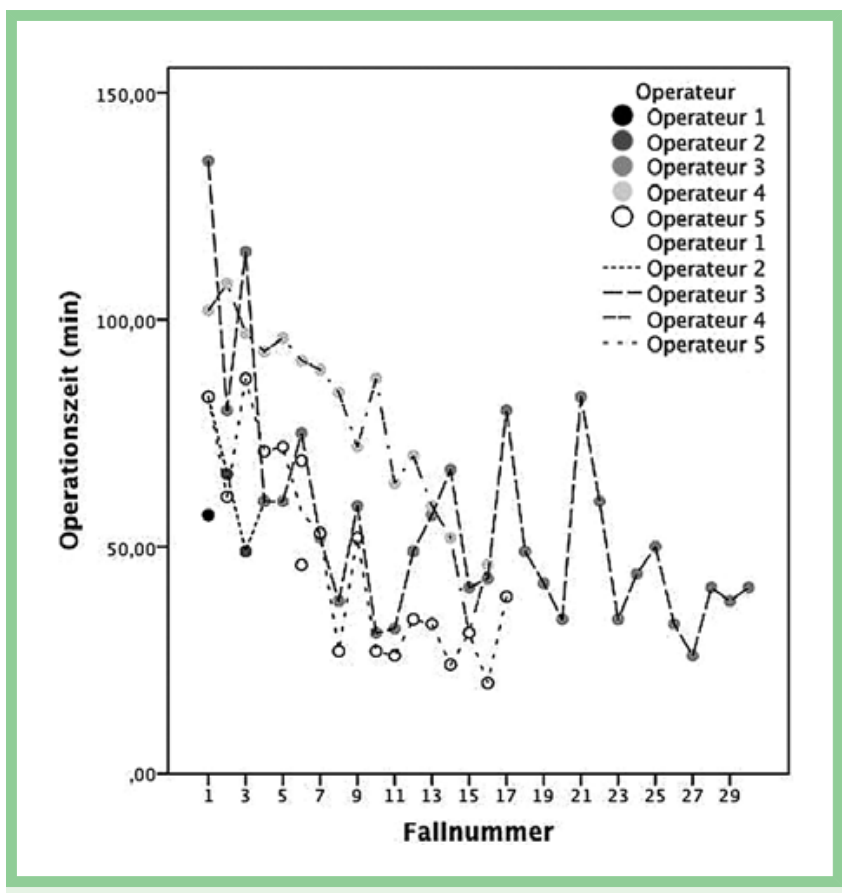

Abb.2 Lernkurve der Single-Port-Methode aufgeschlüsselt für die einzelnen Operateure. Nach einem steilen Abfall erreicht die Kurve nach etwa den ersten zehn Fällen ein Plateau. 
stumpfinsuffizienz, welche mittels ERC und Stenteinlage behandelt wurde. Ein weiterer Patient entwickelte einen Abszess im Gallenblasenbett. Dieser wurde CT-gesteuert drainiert. Zudem kam es zu einer Nachblutung aus dem Nabel, welche mittels Umstechung therapiert wurde. In nur einem Fall $(1,4 \%)$ war eine Revision notwendig: Hierbei handelte es sich um eine Blutung aus dem Omentum majus, welche laparoskopisch mittels Clippung beherrscht werden konnte. Des Weiteren traten drei Harnwegsinfekte als nicht-chirurgische Komplikationen auf. Sieben Patienten, welche mittels Standard-4-Port-Verfahren operiert wurden, erlitten postoperative Komplikationen, davon waren vier chirurgische Komplikationen ( $\bullet$ Tab.3). Auch hier war lediglich in einem Fall aufgrund einer Blutung aus dem Omentum majus eine operative Revision notwendig. Damit ergaben sich keine Unterschiede bezüglich der Raten postoperativer Komplikationen in beiden Gruppen ( $p=0,782$, Chi-Quadrat 0,077). Einen Wundinfekt sahen wir bei keinem Patienten.

\section{Diskussion}

$\nabla$

Der eindeutige Vorteil der Single-Port-Methode ist das bessere kosmetische Ergebnis mit nur einer Narbe, welche im Nabel verschwindet. Mögliche Vorteile wie ein reduzierter Schmerzmittelbedarf [19], weniger Wundinfekte und kürzere Krankenhausaufenthalte werden in der Literatur kontrovers diskutiert [20, 21]. Neben den möglichen Vorteilen werden jedoch auch Bedenken hinsichtlich der Sicherheit im Calot'schen Dreieck bei der Single-
Port-Methode geäußert [22]. Weiterhin ist es auch denkbar, dass durch die größere transumbilikale Inzision beim Single-Port-Vorgehen die Rate an Narbenhernien steigt [23].

Für eine Methode, welche einen rein kosmetischen Vorteil birgt, dürfen Sicherheitseinbußen selbstverständlich nicht akzeptiert werden. Daher ist eine genaue Herausarbeitung von Vorteilen und Risiken der Single-Port-Methode im Vergleich zur 4-PortMethode von immenser Wichtigkeit.

Im Zeitraum von Januar 2009 bis Dezember 2010 wurden in der Landkreis Mittweida Krankenhaus gGmbH 364 laparoskopische Cholezystektomien durchgeführt. Davon wurden 69 Patienten mit der Single-Port-Methode operiert. Für die laparoskopische Cholezystektomie sind ASA-Stadium und Notfalleingriffe Risikofaktoren für das Auftreten von Komplikationen [24]. In unserer Fall-Kontroll-Studie fanden sich keine signifikanten Unterschiede bezüglich des ASA-Stadiums, des Alters und der Rate intraabdomineller Voroperationen. Notfalleingriffe wurden nicht eingeschlossen. Um einen Operateur-spezifischen Bias zu vermeiden, wurden die Schnitt-Naht-Zeiten nach Operateur aufgeschlüsselt. Wir können in unserer Studie eindeutig zeigen, dass keine Unterschiede bezüglich intra- oder postoperativer Komplikationen beider Gruppen vorliegen. Im Falle einer „schwierigen Anatomie“ kann bessere Übersicht durch das Einbringen eines zusätzlichen Trokars gewonnen werden, da hierdurch die Triangulation im Calotschen Dreieck erleichtert wird. Dabei war jedoch nur in einem Fall eine Konversion zum 4-Port-Vorgehen notwendig. Auch bezüglich der Rate operativer Revisionen zeigen sich keine Unterschiede zwischen den beiden Gruppen (1,4\%).

\begin{tabular}{|c|c|c|c|c|}
\hline & & Single Port $(n=69)$ & 4-Port $(n=69)$ & $\mathbf{p}$ \\
\hline \multicolumn{2}{|l|}{ Alter (Jahre) } & $52,4 \pm 1,7$ & $54,9 \pm 1,7$ & 0,3 \\
\hline \multirow[t]{2}{*}{ Geschlecht } & männlich & $15,9 \%$ & $30,4 \%$ & 0,004 \\
\hline & Weiblich & $84,1 \%$ & $69,6 \%$ & \\
\hline \multicolumn{2}{|c|}{ abdominelle Voroperationen } & $8,7 \%$ & $7,2 \%$ & 0,75 \\
\hline \multirow[t]{3}{*}{ ASA } & 1 & $24,6 \%$ & $23,2 \%$ & 0,98 \\
\hline & 2 & $58,0 \%$ & $59,4 \%$ & \\
\hline & 3 & $17,4 \%$ & $17,4 \%$ & \\
\hline
\end{tabular}

Tab. 1 Demografische Daten der Patienten, welche in die Studie eingeschlossen wurden. Für metrisch skalierte Daten ist der Mittelwert \pm der Standardfehler des Mittelwertes angegeben.

Tab.2 Intraoperative Komplikationen und deren Therapie in der SPA-Gruppe, verglichen mit der 4-Trokar-Gruppe.

\begin{tabular}{|c|c|c|c|}
\hline \multicolumn{2}{|l|}{ Single Port } & \multicolumn{2}{|l|}{ 4-Port } \\
\hline Komplikation & Therapie & Komplikation & Therapie \\
\hline $3 \times$ Blutung aus dem Leberbett & Elektrokoagulation & $\begin{array}{l}3 \times \text { Blutung aus dem Leberbett } \\
1 \times \text { Blutung aus dem Omentum } \\
\text { majus }\end{array}$ & $\begin{array}{l}\text { Elektrokoagulation } \\
\text { Blutstillung mittels Clip }\end{array}$ \\
\hline $\begin{array}{l}\text { intraoperative Komplikations- } \\
\text { therapie } 4,3 \%\end{array}$ & & $\begin{array}{l}\text { intraoperative Komplikationsrate } \\
5,8 \%\end{array}$ & \\
\hline
\end{tabular}

Tab.3 Postoperative chirurgische Komplikationen und deren Therapie in der SPA-Gruppe, verglichen mit der 4-Trokar-Gruppe.

\begin{tabular}{|c|c|c|c|}
\hline \multicolumn{2}{|l|}{ Single Port } & \multicolumn{2}{|l|}{ 4-Port } \\
\hline Komplikation & Therapie & Komplikation & Therapie \\
\hline $\begin{array}{l}1 \times \text { Blutung aus dem Hautschnitt } \\
1 \times \text { Abszess im Gallenblasenbett } \\
1 \times \text { Zystikusstumpfinsuffizienz } \\
1 \times \text { Nachblutung aus dem Omen- } \\
\quad \text { tum majus }\end{array}$ & $\begin{array}{l}\text { Umstechung } \\
\text { CT-gestützte Dränage } \\
\text { ERC und Stent } \\
\text { Relaparoskopie und Blutstillung } \\
\text { mit Clip }\end{array}$ & $\begin{array}{l}2 \times \text { Blutung aus dem Hautschnitt } \\
1 \times \text { schwere Paralyse } \\
1 \times \text { Trokarhernie } \\
1 \times \text { Nachblutung aus dem Omentum } \\
\text { majus }\end{array}$ & $\begin{array}{l}\text { Umstechung } \\
\text { medikamentöse Darmstimulation } \\
\text { Herniotomie } \\
\text { Relaparoskopie und Blutstillung } \\
\text { mit Clip }\end{array}$ \\
\hline $\begin{array}{l}\text { Rate postoperativer chirurgischer } \\
\text { Komplikationen } 5,7 \% \\
\text { Revisionsrate } 1,4 \%\end{array}$ & & $\begin{array}{l}\text { Rate postoperativer chirurgischer } \\
\text { Komplikationen } 5,7 \% \\
\text { Revisionsrate } 1,4 \%\end{array}$ & \\
\hline
\end{tabular}


Durch das moderne wirtschaftliche Denken in der Patientenversorgung sind verlängerte Operationszeiten und eine Verlängerung der Krankenhausaufenthaltsdauer nicht mehr akzeptabel. In unserer retrospektiven Fall-Kontroll-Studie betrug die mittlere Schnitt-Naht-Zeit für die Single-Port-Gruppe $58,8 \mathrm{~min}$. Unsere Daten zeigen eindeutig, dass die Einführung der SPA-Cholezystektomie keinesfalls eine Verlängerung der Operationszeiten und der Krankenhausaufenthaltsdauer bedeutet. In unserer Studie sind die Schnitt-Naht-Zeiten der SPA-Gruppe sogar signifikant kürzer als in der 4-Trokar-Gruppe. Dies scheint überraschend, da die SPA-Methode aufgrund des aufwändigeren Zugangsweges und der erschwerten Triangulation per se keine Zeitersparnis offeriert. So sind in den meisten Studien, welche das SPA-Vorgehen mit der 4-Trokar-Methode vergleichen, die Operationszeiten in der Single-Port-Gruppe deutlich länger [14, 19, 25]: In einer Multizenterstudie beträgt die mittlere Schnitt-Naht-Zeit 71 min [26]. Dies entspricht den Schnitt-Naht-Zeiten anderer Untersuchungen $[14,19,25]$. Wir erklären uns die pauschale Zeitersparnis bei der SPA-Methode mit der heterogenen Verteilung der Operateure in den beiden Gruppen: So wurden die meisten 4-TrokarCholezystektomien von einem Operateur durchgeführt, welcher lediglich eine einzige SPA-Cholezystektomie operierte. Auf der anderen Seite wurde ein großer Teil der SPA-Cholezystektomien von einem Chirurgen operiert, welcher auch in der 4-TrokarTechnik vergleichbar kurze Schnitt-Naht-Zeiten aufwies, in dieser Gruppe jedoch nur einen geringen Anteil operierte. Vergleicht man für jeden Operateur die Schnitt-Naht-Zeiten, so unterscheiden sich die Zeiten für die SPA-Cholezystektomie nicht signifikant von denen des 4-Trokar-Vorgehens. Demzufolge lässt sich schlussfolgern, dass die Schnitt-Naht-Zeiten in unserer Studie nicht vom SPA- oder 4-Trokar-Vorgehen, sondern vom Operateur abhängen.

In dem beobachteten Zeitraum fanden das Triport ${ }^{\circledR}$-System von Olympus und das Gelpoint ${ }^{\circledR}$-System von Applied Anwendung. Beide Systeme unterschieden sich nicht hinsichtlich der SchnittNaht-Zeiten und erwiesen sich in unserer Studie als ebenbürtig. Die in der Literatur geäußerten Bedenken hinsichtlich der Sicherheit der Single-Port-Cholezystektomie lassen sich durch unsere Daten nicht bestätigen: So kam es in keinem Fall zu einer intraoperativen Majorkomplikation. Auch die Raten an Minorkomplikationen waren bei beiden Verfahren vergleichbar.

Obwohl die Möglichkeit einer intraoperativen Cholangiografie auch unter Verwendung der Single-Port-Technik beschrieben ist [27], fanden wir in unserem Patientengut keine Indikation dafür. In Fällen von Unsicherheit im Calotschen Dreieck wurde zum 4-Port-Vorgehen konvertiert.

Betrachtet man die Rate an Narbenhernien in der Single-PortGruppe, so ließ sich keine solche innerhalb des 2-jährigen Follow-ups detektieren. Um diese zu vermeiden, führten wir die Inzision entlang der unteren Nabelzirkumferenz durch und vermieden somit den transumbilikalen Zugang. Bei der Betrachtung der Rate an Narbenhernien sei aber auf die kurze Nachbeobachtungsperiode hingewiesen.

In der Literatur ist eine steile Lernkurve für die Single-PortCholezystektomie beschrieben [28]. So konnten auch wir zeigen, dass unabhängig vom Operateur die Schnitt-Naht-Zeit für die ersten fünf Single-Port-Cholezystektomien länger ist als für die folgenden fünf. Nach zehn Fällen wird ein Plateau erreicht. Dies entspricht den Ergebnissen anderer Autoren [14, 28].

Zusammenfassend konnten wir zeigen, dass das bessere kosmetische Ergebnis der Single-Port-Cholezystektomie nicht mit einer niedrigeren Patientensicherheit erkauft wird.

\section{Zusammenfassung}

$\nabla$

In der vorliegenden Fall-Kontroll-Studie konnten wir zeigen, dass die Single-Port-Cholezystektomie der 4-Port-Methode ebenbürtig ist. In Bezug auf Schnitt-Naht-Zeiten, intraoperative und postoperative Komplikationen sind beide Verfahren gleichwertig. Die mittlere postoperative Krankenhausverweildauer ist in der Single-Port-Gruppe tendenziell kürzer, dies ist jedoch statistisch nicht signifikant.

\section{Interessenkonflikt: Nein}

\section{Literatur}

1 Reynolds W Jr. The first laparoscopic cholecystectomy. JSLS 2001; 5: $89-94$

2 Ahmed K, Wang TT, Patel VM et al. The role of single-incision laparoscopic surgery in abdominal and pelvic surgery: a systematic review. Surg Endosc 2011; 25: 378 - 396 DOI: 10.1007/s00464-010-1208-6

3 Marescaux J, Dallemagne B, Perretta $S$ et al. Surgery without scars: report of transluminal cholecystectomy in a human being. Arch Surg 2007; 142: 823-826 discussion 826-827 DOI: 142/9/823 [pii], 10.1001/archsurg.142.9.823

4 Thele F, Zygmunt M, Glitsch A et al. How do gynecologists feel about transvaginal NOTES surgery? Endoscopy 2008; 40: 576-580 DOI: 10.1055/s-2008-1077379

5 Back M, Nimmesgern T, Langwieler TE. [Single port access laparoscopy: a review of the most recent development in minimally invasive surgery]. Zentralbl Chir 2010; 135: 183 - 187 DOI: 10.1055/s-0029-1224753

6 Navarra G, Pozza E, Occhionorelli S et al. One-wound laparoscopic cholecystectomy. Br J Surg 1997; 84: 695

7 Piskun G, Rajpal S. Transumbilical laparoscopic cholecystectomy utilizes no incisions outside the umbilicus. J Laparoendosc Adv Surg Tech A 1999; 9: 361 - 364

8 Jacob DA, Raakow R. [Transumbilical Single-Port Appendectomy: Initial Experience and Technical Report]. Zentralbl Chir 2011: [Epub ahead of print] DOI: 10.1055/s-0031-1271395

9 Antoniou SA, Pointner R, Granderath FA. Single-incision laparoscopic cholecystectomy: a systematic review. Surg Endosc 2011; 25: $367-$ 377 DOI: 10.1007/s00464-010-1217-5

10 Till H, Wachowiak R, Marinoni $F$ et al. [Laparoendoscopic single site cholecystectomy (LESS) in a 16-year-old girl: the way to go even in pediatric surgery?] Zentralbl Chir 2010; 135: 188 -189 DOI: 10.1055/s0029-1224683

11 Bucher P, Ostermann S, Pugin F et al. Female population perception of conventional laparoscopy, transumbilical LESS, and transvaginal NOTES for cholecystectomy. Surg Endosc 2011; 25: 2308-2315 DOI: 10.1007/s00464-010-1554-4

12 Curcillo PG2nd, Podolsky ER. Re: Single incision multiport laparoendoscopic (SIMPLE) surgery. Surg Endosc 2010; 24: 2076- 2077 author reply 2078-2079 DOI: 10.1007/s00464-010-0894-4

13 Aprea G, Coppola BottazziE, Guida F et al. Laparoendoscopic single site (LESS) versus classic video-laparoscopic cholecystectomy: a randomized prospective study. J Surg Res 2011; 166: e109-e112 DOI: S00224804(10)01809-3 [pii]

14 Ma J, Cassera MA, Spaun GO et al. Randomized Controlled Trial Comparing Single-Port Laparoscopic Cholecystectomy and 4-Port Laparoscopic Cholecystectomy. Ann Surg 2011; 254: 22-27 DOI: 10.1097| SLA.0b013e3182192f89

15 Asakuma M, Hayashi M, Komeda Ket al. Impact of single-port cholecystectomy on postoperative pain. Br J Surg 2011; 98: 991-995 DOI: $10.1002 /$ bjs. 7486

16 Bucher P, Pugin F, Buchs NC et al. Randomized clinical trial of laparoendoscopic single-site versus conventional laparoscopic cholecystectomy. Br J Surg 2011; 98: 1695 - 1702 DOI: 10.1002/bjs.7689

17 Steinemann DC, Raptis DA, Lurje G et al. Cosmesis and Body Image after Single-Port Laparoscopic or Conventional Laparoscopic Cholecystectomy: A multicenter double blinded Randomised Controlled Trial (SPOCC-trial). BMC Surg 2011; 11: 24 DOI: 1471-2482-11-24 [pii], 10.1186/1471-2482-11-24

18 Lai EC, Yang GP, Tang CN et al. Prospective randomized comparative study of single incision laparoscopic cholecystectomy versus conven- 
tional four-port laparoscopic cholecystectomy. Am J Surg 2011; 202: 254-258 DOI: S0002-9610(11)00209-1 [pii]

19 Chang SK, Tay CW, Bicol RA et al. A case-control study of single-incision versus standard laparoscopic cholecystectomy. World J Surg 2011; 35 : 289-293 DOI: 10.1007/s00268-010-0842-4

20 Duron VP, Nicastri GR, Gill PS. Novel technique for a single-incision laparoscopic surgery (SILS) approach to cholecystectomy: single-institution case series. Surg Endosc 2011; 25: 1666 - 1671 DOI: 10.1007/ s00464-010-1374-6

21 Langwieler TE, Nimmesgern T, Back M. Single-port access in laparoscopic cholecystectomy. Surg Endosc 2009; 23: 1138 - 1141 DOI: 10.1007| s00464-009-0389-3

22 Papagoras D, Kanara M, Argiropoulos-Rakas $C$ et al. Single port access laparoscopic cholecystectomy (with video). World J Surg 2011; 35: 235-236 DOI: 10.1007/s00268-010-0678-y

23 Elsey JK, Feliciano DV. Initial experience with single-incision laparoscopic cholecystectomy. J Am Coll Surg 2010; 210: 620-626 DOI: S1072-7515(09)01687-1 [pii], 10.1016/j.jamcollsurg.2009.12.030
24 Jakob J, Hinzpeter M, Weiss C et al. [Evaluation of data on surgical complications after cholecystectomy submitted to a nationwide quality assurance program (BQS) in Germany]. Chirurg 2010; 81: 563-567 DOI: $10.1007 /$ s00104-009-1827-4

25 Khambaty F, Brody F, Vaziri K et al. Laparoscopic versus single-incision cholecystectomy. World J Surg 2011; 35: 967-972 DOI: 10.1007/ s00268-011-0998-6

26 Curcillo PG 2nd. Single-incision laparoscopic right hemicolectomy. $\mathrm{Br} \mathrm{J}$ Surg 2010; 97: 1884 DOI: 10.1002/bjs.7260

27 Bagloo MB, Dakin GF, Mormino LP et al. Single-access laparoscopic cholecystectomy with routine intraoperative cholangiogram. Surg Endosc 2011; 25: 1683 - 1688 DOI: 10.1007/s00464-010-1408-0

28 Solomon D, Bell RL, Duffy AJ et al. Single-port cholecystectomy: small scar, short learning curve. Surg Endosc 2010; 24: 2954-2957 DOI: $10.1007 / \mathrm{s} 00464-010-1070-6$ 